\section{Acknowledgements}

I wish to thank Mr. J. Waddington, University of Saskatchewan photographer, for his help in making the plates and taking most of photographs. Information in this article is from part of a research project financed by the University of Saskatchewan's Institute for Northern Studies and by a National Research Council of Canada grant held by D. M. Lehmkuhl. Preparation of this article was sponsored by the Entomological Society of Saskatchewan.

\section{USEFUL REFERENCES}

Darlington, A. 1968. The Pocket Encyclopaedia Plant Galls. Blandford Press. London. 191 pages

Felt, E. P. 1940. Plant Galls and Gall Makers Comstock Publishing Company. 364 pages Reprinted 1965 by Hafner Publishing Compan Inc. New York.

Hutchins, R. E. 1969. Galls and Gall Insects. Dodd Mead and Company, New York. 128 pages.

Mani, M. S. 1964. Ecology of Plant Galls. W. Junk Publishers. The Hague, Netherlands. 434 pages

Shorthouse, J. D. 1973. The Insect Communit Associated With Rose Galls of Diplolepis polit (Cynipidae, Hymenoptera). Quaest. ent. In pres

\title{
31st ANNUAL \\ SASKATCHEWAN CHRISTMAS BIRD COUNT 1972
}

\section{Compiled by MARY I. HOUSTON*}

Perhaps the generally mild weather over the count period was an encouraging fac tor in the record high of 46 localities reporting for the Christmas Bird Census thi year. Fifty-five species were seen on count days with an additional 6 species seer during the count period. A Ruby-crowned Kinglet identified at Biggar by Dor Renaud adds a new species to the all time list, bringing the total to 115 species witl 6 additionals.

Especially interesting records included the two Hooded Mergansers seen at Gar diner Dam by the Renauds. This species has only been recorded once previousl when in 1960 Ross Lein and Darrel Carlson saw three at Estevan. A Borea Chickadee seen at Wauchope by Dale Hjertaas during the count period wa unusually far south. Unfortunately, the only Gyrfalcon seen during the period (a Belle Plaine, Dec. 20, by Bob McCall) was not in a bird count. There was only Bald Eagle report this year, at Squaw Rapids. White-breasted Nuthatches seeme more common than usual, being reported from five localities, while Red-breaste Nuthatches were reported from only one locality. Snowy Owls seemed to be in large numbers (e.g., 13 at Harris) in many of the 21 areas in which they were seen. On th other hand, Gray Partridge, which a number of counters felt had decreased greatl in number, were seen in 25 areas, though probably in lower numbers than usual i each area. Ravens were reported from more southern localities (Broadview, Harri: Raymore, Saskatoon, Spirit Lake) than usual.

Special commendation goes to Wayne and Don Renaud who arranged five count and Wayne Harris who arranged four.

BANGOR, Dec. 31 ; temp. $-20^{\circ}$, sunny; 8 species, 54 individuals. - Mrs. A. Thompson.

BIGGAR, Dec. 16; temp. 20, light cloud, wind light, 3 in. snow: 11 species, 2699 individuals. (Add: Snow Bunting, 1500, Dec. 22). - Don Renaud, Wayne Renaud (compiler).

*863 University Drive, Saskatoon.
BIRCH HILLS, Dec. 27; $9 \mathrm{mi}$. by car in hour, temp. $20^{\circ}$, overcast, calm, 8 in. snow species, 446 individuals. (Add: Ruff Grouse, 4, Common Raven, 9, Eveni Grosbeak, 9). - Moe Mareschal.

BORDEN, Dec. 30; 2 mi. on foot in 1 ho and $79 \mathrm{mi}$. by car in $5-1 / 2 \mathrm{hrs}$, temp. $-17^{\circ}$ $2^{\circ}$, wind $E 8$ to $0 \mathrm{mph}$, cloudy with aft noon fog; 13 species, 1442 individuals. and Vic Harper, John Shadick, Stan Shadi (compiler). 


\begin{tabular}{|c|c|c|c|c|c|c|c|c|c|c|c|c|c|c|c|}
\hline & $\dddot{\pi}$ & $\ddot{n}$ & 急 & 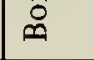 & 公 & U & อ & $\bar{\theta}$ & 可 & 凷 & 里 & 䒰 & ज़ & 3.9 & 心 \\
\hline \multicolumn{16}{|l|}{ Mute Swan } \\
\hline \multicolumn{16}{|l|}{ Canada Goose } \\
\hline Mallard & & & & & & & & & & & & & 235 & & \\
\hline \multicolumn{16}{|l|}{ Redhead } \\
\hline \multicolumn{16}{|l|}{ Canvasback } \\
\hline Lesser Scaup & & & & & & & & & & & & & 1 & & \\
\hline Common Goldeneye & & & & & 6 & & & & & & & & 12 & & \\
\hline Hooded Mergr. & & & & & & & & & & & & & 2 & & \\
\hline \multicolumn{16}{|l|}{ Goshawk } \\
\hline Golden Eagle & & & & & 2 & & & 1 & & & & & & & \\
\hline \multicolumn{16}{|l|}{ Bald Eagle } \\
\hline \multicolumn{16}{|l|}{ Marsh Hawk } \\
\hline \multicolumn{16}{|l|}{ Prairie Falcon } \\
\hline \multicolumn{16}{|l|}{ Pigeon Hawk } \\
\hline Ruffed Grouse & & & & & 1 & & & & & & & & & & \\
\hline Sharp-tailed Grouse & 3 & & & 15 & 4 & 8 & 1 & & & 8 & & 4 & & 35 & \\
\hline \multicolumn{16}{|l|}{ Ring-neck Pheasant } \\
\hline Gray Partridge & 9 & 7 & & 7 & & & & 11 & & & & 22 & & 2 & 10 \\
\hline \multicolumn{16}{|l|}{ Am Coot } \\
\hline Rock Dove & & 71 & & & 13 & & & & & & 2 & 15 & 12 & 11 & 6 \\
\hline Great-horned Owl & & & & & & & & & & & 2 & & & & \\
\hline Snowy Owl & & 1 & & & 1 & & 1 & & & & & & & 2 & \\
\hline Short-eared Owl & & & & & 1 & & & & & & & & & & \\
\hline \multicolumn{16}{|l|}{ Pileated Woodpecker } \\
\hline Hairy Wdpkr. & 1 & & & & 1 & & 1 & & 1 & & 1 & & & & 1 \\
\hline Downy Wdpkr. & 1 & & & 1 & & & & & & 1 & 1 & & & & \\
\hline N.3-t. Wdpkr. & & & & & & & & & 1 & & & & & & \\
\hline Horned Lark & & & & 21 & & & & 14 & & & 3 & & & & \\
\hline Gray Jay & & & & & & & & & 3 & & & & & & \\
\hline Blue Jay & & & & & 2 & & & & & & & & & & \\
\hline Black-billed Magpie & 2 & 24 & 2 & 24 & 17 & 31 & 22 & 7 & & 7 & 53 & 9 & 12 & 10 & 2 \\
\hline Common Raven & & & & & & & & & 1 & 4 & & & & & \\
\hline Bl-cap Chickadee & 4 & & 2 & 2 & 19 & & 3 & 1 & 2 & 6 & 11 & 3 & 1 & & 2 \\
\hline \multicolumn{16}{|l|}{ Boreal Chickadee } \\
\hline W-b. N'hatch & & & 1 & & 1 & & & & & & & & & & \\
\hline R-b. N'hatch & & & & & & & & & & & & & & & \\
\hline Robin & & & & & & & & & & & & & & & \\
\hline R-c Kinglet & & 1 & & & & & & & & & & & & & \\
\hline Boh. Wax wing & & 183 & 78 & 5 & 68 & & & & & & 42 & & & & \\
\hline Ced. Waxwing & & & & & & & & & & & & & & & \\
\hline Northern Shrike & & & & & 2 & & & & & & & & & & \\
\hline Starling & & & & & & & & & & 7 & & & & & \\
\hline House Sparrow & 20 & 2332 & 363 & 115 & 186 & & 100 & 90 & & 21 & 80 & 250 & 104 & 100 & 300 \\
\hline Rusty Blkbd. & & & & & & & & & & & & & & & \\
\hline Ev. Grosbeak & 14 & 14 & & 48 & 28 & & & & & & 57 & & & & \\
\hline Pine Grosbeak & & 60 & & 21 & 20 & & 14 & 1 & & & 11 & 13 & & & 1 \\
\hline Hoary Redpoll & & & & 10 & & & & & & & & & & & \\
\hline Common Redpoll & & 4 & & 1164 & 86 & 35 & 50 & & 1 & & 1 & & & & \\
\hline Pine Siskin & & & & & & & & & & & & & & & \\
\hline Red Crossbill & & & & & & & & & & & & & & & \\
\hline Slate-colored Junco & & 2 & & & & & & & & & & & & & \\
\hline Tree Sp. & & & & & & & & & & & & & & & \\
\hline Harris' Sp. & & & & & & & & & & & & & & & \\
\hline Lapl. Longspur & & & & & & & & & & & & & & & \\
\hline Snow Bunting & & & & 9 & 21 & & 35 & 1 & & 51 & 19 & & 10 & & \\
\hline
\end{tabular}


BROADVIEW, Dec. 17;95 mi. by car in 7 hrs., temp. $26^{\circ}$, calm; 19 species, 479 individuals plus 1 unidentified accipiter. (Add: Horned Lark, 7, Dec. 31: Common Raven, 2 , Dec. 31). - David Chaskavich, Donald Weidl.

CRAVEN, Dec. 26; $25 \mathrm{mi}$. by car in $2-1 / 2$ hrs. and $1 / 2$ hour on foot, temp. $40^{\circ}$ clear, no wind. 2 in. snow: 3 species, 74 individuals. - Tom Donald, Bob Rafuse.

DALMENY, Dec. 26; 4 hrs. on foot, temp. $17^{\circ}$, partly cloudy light $\mathrm{E}$ wind; 9 species, 227 individuals. - Brian Gilbert (compiler) and Loyd Sperling.

DILKE, Dec. 3I:34 mi. by car in $2 \mathrm{hrs}$ and 3 hrs. about the yard, temp. $-3^{\circ}$ to $3^{\circ}$, overcast, wind $\mathrm{N} 5 \mathrm{mph}, \mathrm{I} 2 \mathrm{in}$. snow; 8 species, 126 individuals. (Add: Snowy Owl, 1, Dec. 19: Common Redpoll, 2, Dec. 16). - Boswell Belcher (compiler), Margaret Belcher, Mr. and Mrs. S. R. Belcher.

ELDORADO, Dec. 28; within $1 / 2$ mile of house, temp. $5^{\circ}$ to $10^{\circ} ; 6$ species, 9 individuals. - Mrs. E. A. Middleton.

ENDEAVOUR, Dec. 26; around house, and 10 miles by car, temp. $-10^{\circ}$ to $10^{\circ}$, cloudy, clearing at mid day, calm; 8 species, 105 individuals, (Add: Great Horned Owl, 1, Dec. 24; Gray Jay, 2, Dec 24; Common Redpoll, 7, Dec. 25.) - William Haras.

FEUDAL, Dec. 26 ; temp. $40^{\circ}$, partly cloudy, wind NW 15-20 mph, 3 in. snow; 13 species, 283 individuals. - Don and Wayne Renaud.

FILLMORE, Dec. 26; $6 \mathrm{mi}$. by car, 2-1/2 mi. on foot, temp. $20^{\circ}$ to $30^{\circ}$, sunny, wind light, 6-7 in. snow; 7 species, 316 individuals. (Add: Snowy Owl, 1, Dec. 24; Common Redpoll, 11, Dec. 24). - Mrs. Nick Bogdan (compiler), Nick Bogdan, Ken Elder, Gilbertha Liebelt, Larry and Marie Wiggins.

GARDINER DAM, Dec. 20; temp $20^{\circ}$, clear, wind NW.0-5 mph, 3 in. snow; 9 species, 389 individuals. - Don, Harvey and Wayne Renaud.

GLAMIS-BARBER LAKE, Dec. 26; $12 \mathrm{mi}$. by car and $3 \mathrm{mi}$. on foot in $3 \mathrm{hrs}$., temp $35^{\circ}$ to $41^{\circ}$, wind SW 10-15 mph, 5-6 in. snow. overcast: 6 species, 170 individuals. (Add: Bohemian Waxwing 15, Dec. 24; Snow Bunting, 5, Dec. 24). - Brian and Grev Jones (compilers), Chester Walker.

GRENFELL, Dec. 24; $6 \mathrm{mi}$. by car and around yard, temp. $10^{\circ}, 3$ in. snow: 6 species, 322 individuals. (Add: Ruffed Grouse, I, Dec. 20: Sharp-tailed Grouse, 6, Jan. 1: Snow Bunting. 15, Jan. 2). - Betty and John Hubbard.
MARRIS, Dec. 17; temp. $18^{\circ}$ to $12^{\circ}$, mostly clear, wind $\mathrm{E} 0$ to $5 \mathrm{mph}, 4$ in. snow; 25 species, 3405 individuals. (Add: ?falcon Dec. 25; Northern Shrike, 1, Dec. 31; Slate. coloured Junco, 1, Dec. 16). - Ron Bobowski, Bob Godwin, Bernie Gollop Madelaine Gollop. Wayne Harris, Dale Hjertaas, David, Mary and Stuart Houston Sheila Lamont, Ken Lunbis, Don Renaud Wayne Renaud (compiler), Stan Shadick, Jin Wedgwood.

HEPBURN, Dec. 22; temp. 10: 3 species 4 individuals. $-\mathrm{P}$. Siemens.

KENASTON, Dec. 17: 1 hour on foot an while working about yard and $13 \mathrm{mi}$. by car temp. $15^{\circ}$, wind light; 7 species, 88 in dividuals. (Add: Horned Lark, 7, Dec. 19 Black-capped Chickadee, I Dec. 24 Bohemian Waxwing, 12, Dec. 18: Evenin Grosbeak, 7, Dec. 26: Pine Grosbeak, 3, De 27: Common Redpoll, 50, Dec. 28: Sno Bunting, 200, Dec. 22). - P. Lawrenc Beckie.

KINDERSLEY, Dec. 24: $29 \mathrm{mi}$. by car an $1 / 4 \mathrm{mi}$. on foot around yards, temp. $2^{\circ}$, win light. scattered cloud: 7 species, 190 in dividuals. (Add: Sharp-tailed Grouse, 4, De 31; Bohemian Waxwing, 14, Dec. 19). Jean and Lane Harris, Edith Gardiner.

KUTAWAGAN LAKE, (centered $12 \mathrm{mi}$. i of Semans), Dec. $31 ; 52 \mathrm{mi}$. by car in $3 \mathrm{hr}$ and $3-1 / 4 \mathrm{mi}$. on foot in $1-1 / 2$ hrs. tem $-10^{\circ}$ to $1^{\circ}$, foggy, clearing, wind NE 0 . mph. 7 in. snow; 5 species, 187 individua (Add: Gray Partridge, 5, Dec. 26; Great Ho ned Owl, 2, Dec. 22; Horned Lark, 1, Dr 16). - Wayne Harris.

LAST MOUNTAIN LAKE, (centered 8 n SW of Nokomis), Jan. 1; $46 \mathrm{mi}$. by car in hrs and $3 \mathrm{mi}$. on foot in $1-1 / 2 \mathrm{hrs}$, temp. 1 to $15^{\circ}$, sunny becoming overcast, wind 10-15 mph, 9 in. snow; 9 species 595 dividuals. (Add: Common Redpoll, 3, D 27). - Wayne Harris.

LUSELAND, Dec. 24; $130 \mathrm{mi}$. by car in hrs and $5 \mathrm{mi}$. on foot in $2 \mathrm{hrs}$, temp. $10^{\circ}$ $15^{\circ}$, sunny, wind NW $0-5 \mathrm{mph}, 2-3$ in. sno 16 species 445 individuals. (Add: Horn Lark, 3, Dec. 23: Evening Grosbeak, 8, D 27). - Kerry and Kim Finley, Bill Holto MELVILLE, Dec. 26; around the city; species, 86 individuals. (Add: Downy Woc pecker.) - Kay Anweiler.

MONTMARTRE, Dec. 23; $14 \mathrm{mi}$. on foot 6 hrs., temp. $0^{\circ}$ to $10^{\circ}$, overcast then cleari wind NW 5 mph; 7 species, 183 individue (Add: Sharp-tailed Grouse, 21, Dec. Rock Dove, 1, Jan. 1: Snowy Owl, 1, D 16; Rusty Blackbird, 4, Dec. 30). - K Langelier. 


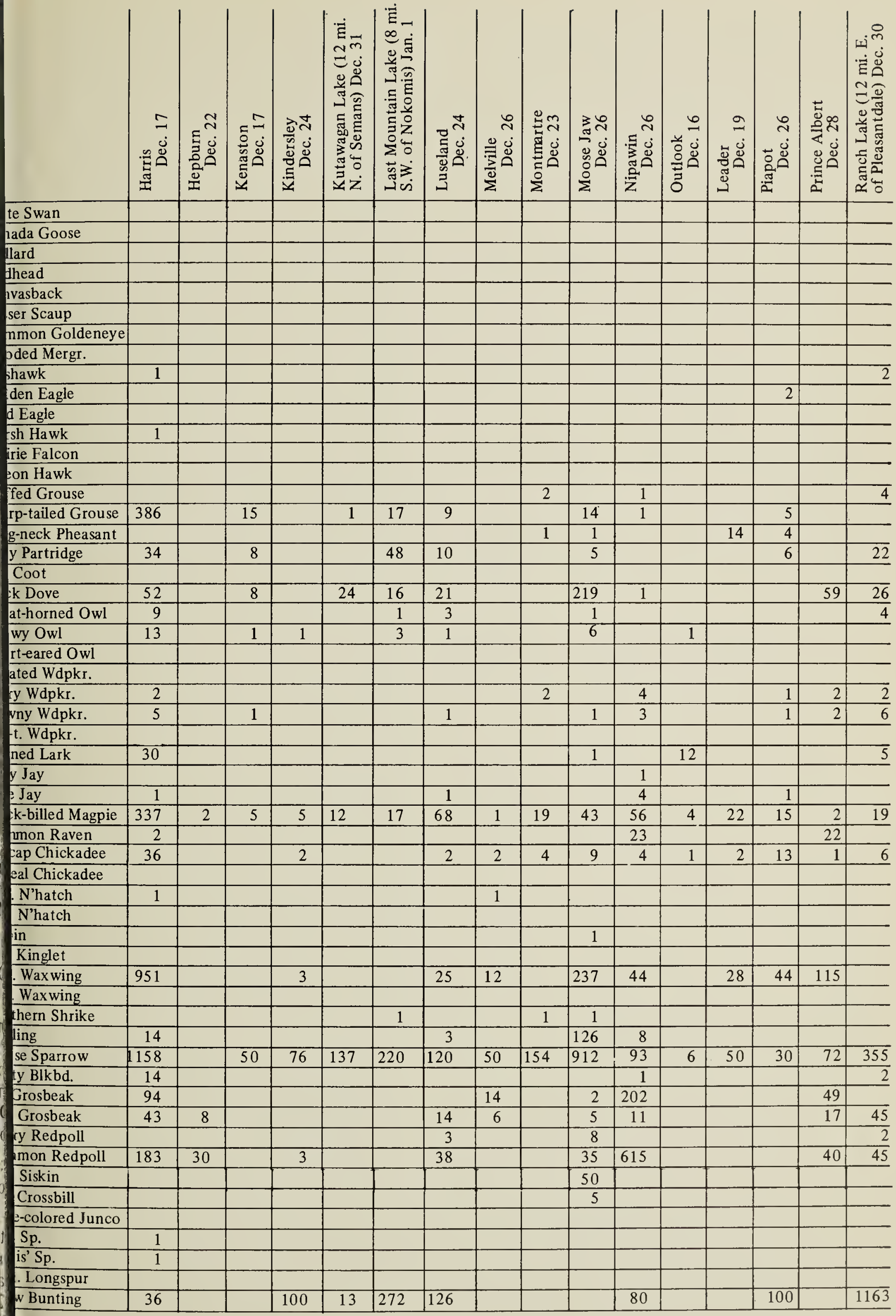


MOOSE JAW, Dec. 26; $112 \mathrm{mi}$. by car and 6 mi. on foot, temp. $38^{\circ}$, sunny, wind SW 10 $15 \mathrm{mph}$, little snow; 21 species, 1682 individuals (Add: Golden Eagle, 1, Dec. 31: Pigeon Hawk, 1, Dec. 31). - Anna Davis Doug Francis, Ruth Hillings, Pat Kern, Leith Knight, (compiler) Moray Lewis, Molly Ritchie, Dave Robinson, Alice and Gordon Silversides, Inez Simmons, Dick Springett and Jean Thomson.

NIPAWIN, Dec. 26; temp. $20^{\circ}$, no wind: 18 species, 1152 individuals. - Terry Gingard, Vern Gunnlaugson, Gladys and Stan Riome (compilers), Mrs. Max Robin.

OUTLOOK, Dec. 16; 60. by car, temp $12^{\circ}$, sunny, light wind, 5 in. snow; 5 species, 24 individuals (Add: Bohemian Waxwing, 80, Dec. 27). - Harold Kvinge.

LEADER, Dec. 19; 2 hours about the ranch, temp $40^{\circ}$, clear, 3 in. snow; 5 species, 116 individuals. (Add: Rock Dove, 8, Dec. 24). Daisy Meyers.

PIAPOT, Dec. 26; temp. 30\%; 12 species, 222 individuals. - Jim Bennetto, Betty Mann, Robert Mann, Don Pearce, Helen Schuler, Lyall Wagner.

PRINCE ALBERT, Dec. 28; 11 species, 381 individuals. (Add: Ruffed Grouse, 1, Dec. 23). - Mr. and Mrs. Ansgar Aschim, Mr. and Mrs. Bert Gordon, David Surkan.

RANCH LAKE (centered 12 mi. E. of Pleasantdale), Dec. 30; $107 \mathrm{mi}$. by car in 6 hrs. and 4-1/2 mi. on foot in 2 hrs., temp. $-14^{\circ}$ to $-4^{\circ}$, foggy, wind SW $0-10 \mathrm{mph}, 11$ in. snow; 16 species, 1708 individuals. (Add: Common Raven 1, Dec. 22; Snowy Owl, 1, Dec. 25). - Greta Harris, Wayne Harris (compiler), Elaine Rice.

RAYMORE, Dec. $26 ; 128 \mathrm{mi}$. by car in 8 hrs. and 2 mi. on foot in 1 hour, plus observations at feeder, temp. $21^{\circ}$ to $38^{\circ}$, overcast, wind SW 0-13 mph, 7 in. snow; 18 species, 2337 individuals, (Add: Snowy Owl, 1, Dec. 22; Evening Grosbeak, 2, Jan. 1). Charles, Greta and Wayne Harris.

REGINA, Dec. 26; $509 \mathrm{mi}$. by car in 46 hours and $54 \mathrm{mi}$. on foot in $31 \mathrm{hrs}$, temp. $29^{\circ}$ to $38^{\circ}$, high clouds clearing later, wind SW 5-15 mph., 3 in. snow; 32 species, 5395 individuals. (Add: Goshawk, 1, Dec. 20; Barred Owl, 1, Dec. 31; Yellow-shafted Flicker, 1, Dec. 21; Common Crow, 1, Dec. 23). - Jessie Bailey, Margaret Belcher, Al and Betty Binnie, Tom Burns, Betty Cruickshank, Ken Dickson, Dick DuWors, Lucy Eley, George Ferguson, Wayne Gemmell, Doug Gilroy, Jim Hines, Roger and Pat Howard, Jim and Shirley Jowsey, Darlene Kauk, Bob Kreba, George Ledingham, Sandra McNeil, Helen Morrison. Eric Olsen,
Patrick Pettit, John Pilling, Connie Pratt Brian Rainey, Maureen Rever, Joe Roberts Paul Rump, R. Lorne Scott (compiler), Joh and Carol Spencer, Frank Switzer, Doroth: Tegart, Elisabeth Wagner, Jeanie Wagner Janie Wilhelm, Rita Wilhelm, Don Young.

ROSETOWN, Dec. 19; temp $20^{\circ}$, light cloud wind light, 3 in. snow; 6 species, 457 in dividuals. (Add: Snowy Owl, 1, Dec. 27). Annie Harvey, Wayne Renaud.

SASKATOON, Dec. 26: $224 \mathrm{mi}$. by car in 2 hrs., and $55 \mathrm{mi}$. on foot in $43 \mathrm{hrs}$., temp. 20 to $34^{\circ}$, wind SW 3-22 mph, 2 in. snow; 2 species, 6161 individuals. (Add: Short-eare Owl, 1, Dec. 20; Yellow-shafted Flicker, Dec. 27; Common Raven, 2, Dec. 24; Pir Siskin, 7, Dec. 27; Red Crossbill, 15, De 20; White-winged Crossbill, 2, Dec. 22). Joan Ashenhurst, Bob, Jeff and Joan Besan Bernard, Ed, Raymond and Timothy Bish Andre Bouthilette, Don Buckle, Ric Buchner, Pern Cordery, Joseph E. Daly, R: and Molly Denson, John and Betty Gerrar Bernie and Madeleine F. Gollop, Jack an Louise Greaves, Scott Hale, Danny Heffe nan, Stuart, Mary, Dave and Don Housto Dr. C. J. Houston, Mary and Tom Kearne Carolyn and Colin Kindrachuk, Do Elizabeth, Joanne, Margaret and Norme McRobbie, Greg Michalenko, Alan Moulin, Arnold Nijssen, Stuart Rasmusse Adam Schmidt, David Schmidt, John an Stan Shadick, Les Shand, J. A. Slimmo Alan R. Smith, Gary Smith, Peter Tassie, W. A. Whitfield, Elmer and Winnie Wrigh SOMME, Dec. 26; $25 \mathrm{mi}$. by car and on for temp. $15^{\circ}: 14$ species, 92 individuals. (Ad Evening Grosbeak, 7, Dec. 24; Hoary Re poll, 1, Dec. 25: Snow Bunting, 80, Dec. 2 - B., D., E. and R. Hooper.

SORENSON BEACH (Last Mountain Lak Dec. $25 ; 6 \mathrm{mi}$. in 5 hours on foot, temp 1 to $20^{\circ}$, wind NW 5-15 mph, 2-3 in. snow; species 123 individuals. - Dr. C. H. Ficl Dr. Hanna Ueberschar, Dr. Heinz Ueb schar.

SPIRIT LAKE, Dec. 26: $57 \mathrm{mi}$. by car in hrs and $2 \mathrm{mi}$. on foot in $1-1 / 2 \mathrm{hrs}$, temp. to $28^{\circ}$, mostly clear, wind light, 8 in. snd 15 species, 260 individuals. (Add: Sno Owl, 1, Dec. 18; Common Raven, 11, D 18: Bohemian Waxwing, 9, Dec. 23: N thern Shrike, 1, Dec. 27; Evening Grosbe 3, Dec. 25). - Bill and Joyce Anaka, M Gunn.

SPRING VALLEY, Dec. 31; 34-1/2 mi. car and $13 \mathrm{mi}$. by skidoo and $2-1 / 2 \mathrm{mi}$. foot in $7-1 / 2$ hrs., temp. $3^{\circ}$, sunny and $\mathrm{ca}^{\circ}$ then overcast with wind 25-30 mph, 4 snow; 10 species, 943 individuals. (A Golden Eagle, 1, Dec. 19; Prairie Falcon 


\begin{tabular}{|c|c|c|c|c|c|c|c|c|c|c|c|c|c|c|c|c|}
\hline & 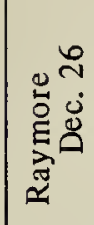 & 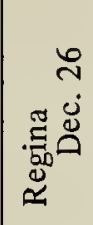 & 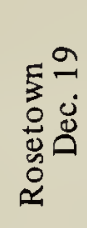 & 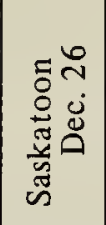 & 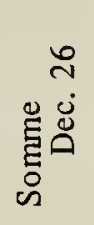 & 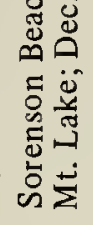 & 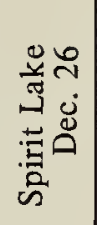 & 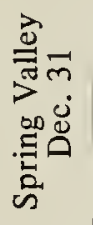 & 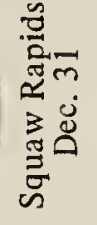 & 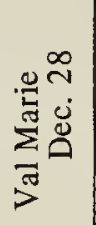 & 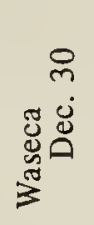 & 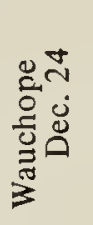 & 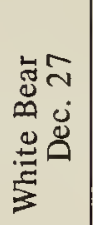 & 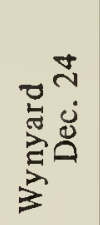 & 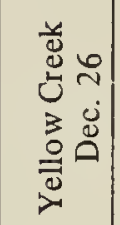 & $\begin{array}{l}\stackrel{n}{\Xi} \\
\vdots \\
0 \\
\dot{0} \\
\dot{0} \\
\dot{z}\end{array}$ \\
\hline Swan & & 3 & & & & & & & & & & & & & & 1 \\
\hline ada Goose & & 450 & & & & & & & & & & & & & & $\frac{1}{1}$ \\
\hline ard & & 350 & & 35 & & & & & & & & & & & & 3 \\
\hline head & & 1 & & & & & & & & & & & & & & $\overline{1}$ \\
\hline vasback & & 1 & & & & & & & & & & & & & & $\overline{1}$ \\
\hline er Scaup & & 7 & & & & & & & & & & & & & & 2 \\
\hline imon Goldeneye & & 4 & & 65 & & & & & 7 & & & & & & & 5 \\
\hline ded Mergr. & & & & & & & & & & & & & & & & $\overline{1}$ \\
\hline hawk & & & & 3 & 2 & 1 & 1 & & & & & & & & & 6 \\
\hline den Eagle & & & & & & & & & & & & & & & & 3 \\
\hline 1 Eagle & & & & & & & & & 1 & & & & & & & 1 \\
\hline sh Hawk & & & & & & & & & & & & & & & & 1 \\
\hline rie Falcon & & 1 & & & & & & & & & & & & & & 1 \\
\hline on Hawk & & 1 & & 6 & & & & & & & & & & & & $\overline{2}$ \\
\hline fed Grouse & 1 & 3 & & 6 & & 1 & 1 & & & & & & & & 2 & 10 \\
\hline p-tailed Grouse & 45 & 14 & & 177 & & 6 & 1 & 10 & & & & 11 & & 9 & 3 & 25 \\
\hline -neck Pheasant & & & & 15 & & & & & & 14 & & & & & & 6 \\
\hline Partridge & 19 & 32 & & 66 & 8 & 16 & 12 & 85 & & & & & 18 & & & 22 \\
\hline Coot & & 3 & & & & & & & & & & & & & & $\overline{1}$ \\
\hline k Dove & 105 & 361 & 24 & 678 & & & & 43 & & & & 23 & & 6 & 20 & $\overline{24}$ \\
\hline t-horned Owl & 5 & 5 & & 1 & 1 & 2 & 1 & 1 & & & & & & & 2 & 14 \\
\hline wy Owl & & 5 & & 5 & & & & 1 & & & & & & & & $\overline{14}$ \\
\hline t-eared Owl & & & & & & & & & & & & & & & & 1 \\
\hline ted Wdpkr. & & & & & 1 & & & & & & & & & & & $\overline{1}$ \\
\hline y Wdpkr. & 2 & 2 & & 13 & 2 & & 8 & & & & & 1 & & & & 18 \\
\hline ny Wdpkr. & 1 & 3 & & 12 & 1 & & 9 & & & & 1 & 4 & & 1 & 1 & 21 \\
\hline t. Wdpkr. & & & & & & & & & & & & & & & & 1 \\
\hline ed Lark & & & & 5 & & 9 & & 111 & & & & & & & & 10 \\
\hline Jay & & & & & 6 & & 1 & & & & & & & & & $\overline{4}$ \\
\hline Jay & 1 & & & 14 & 1 & & & & & & 5 & & & & & 9 \\
\hline x-billed Magpie & 80 & 89 & 7 & 288 & 3 & 5 & 22 & 21 & 6 & 6 & & 13 & 19 & 16 & 10 & 44 \\
\hline mon Raven & 1 & & & & 7 & & & & 7 & & & & & & & 8 \\
\hline ap Chickadee & 32 & 51 & & 157 & 4 & 4 & 32 & & & 1 & 12 & 12 & & 6 & 9 & 35 \\
\hline al Chickadee & & & . & & 1 & & & & & & & & & & 1 & $\overline{1}$ \\
\hline N'hatch & & & & & & & 3 & & & & & & & & & 5 \\
\hline N'hatch & & & & 2 & & & & & & & & & & & & 1 \\
\hline n $\quad$ & & & & 7 & & & & & & & & & & & & 2 \\
\hline Kinglet & & & & & & & & & & & & & & & & 1 \\
\hline Waxwing & 23 & 727 & 59 & 1116 & & 8 & & & & 1 & 14 & & & & 20 & 22 \\
\hline Wax wing & & 3 & & & & & & & & & & & & & & 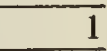 \\
\hline hern Shrike & 1 & & & 4 & & & & & & & & & & & & 6 \\
\hline ing & & 28 & 4 & 16 & & & & & & & & & & & & -8 \\
\hline e Sparrow & 1911 & 3029 & 353 & 2820 & 35 & 35 & 94 & 505 & & 10 & 26 & 254 & & 306 & 500 & 41 \\
\hline y Blkbd. & 2 & 2 & & 6 & & & & & & & & & & & & 6 \\
\hline rosbeak & & 50 & & 116 & & 4 & & & & & 5 & & & 2 & & 15 \\
\hline Grosbeak & 25 & 59 & & 250 & 20 & 32 & 12 & & 11 & 5 & 5 & $\overline{44}$ & & 24 & & 27 \\
\hline y Redpoll & & & & & & & & & & & & & & & & 4 \\
\hline mon Redpoll & 23 & 77 & & 185 & & & 11 & 46 & & 3 & & & 19 & & & 22 \\
\hline Siskin & & 1 & & & & & & & & & & & & & & $\overline{2}$ \\
\hline Crossbill & & 3 & & & & & & & & & & & & & & 2 \\
\hline -colored Junco & & 3 & & & & & & & & & & & & & & 2 \\
\hline \multicolumn{17}{|l|}{ Sp. } \\
\hline s' Sp. & & & & & & & & & & & & & & & & 1 \\
\hline Longspur & & & & 6 & & & & & & & & & & & & $\overline{1}$ \\
\hline Bunting & 60 & 27 & 10 & 87 & & & 52 & 120 & 200 & & 49 & 311 & & 188 & 40 & 26 \\
\hline
\end{tabular}


Dec 23: Sparrow Hawk, I, Dec. 17; Bohemian Waxwing, 4, Dec. 16). - Allan Bogdan, Mr. and Mrs. Nick Bogdan, Gilbertha Liebelt.

SQUAW RAPIDS, Dec. 31: 4 hrs, temp. 5०, heavy hoar frost; 6 species 232 individuals. - David and Stan Riome.

VAL MARIE, Dec. 28; $30 \mathrm{mi}$. by car and 2 mi. on foot, temp. $25^{\circ}$, snowing, no wind, very little snow on ground; 7 species, 40 individuals. (Add: Sharp-tailed Grouse, 6, Dec. 29; Gray Partridge, 9, Dec. 18; Rock Dove, 7, Dec. 30; Great Horned Owl, 1, Dec. 18: Long-eared Owl, 1, Dec. 26; Short-eared Owl, I, Jan. 1: Horned Lark, 30, Dec. 20: Snow Bunting, 300, Dec. 17). - J. David Chandler.

WASECA, Dec. 30; around 2 farm yards and on foot $3 \mathrm{mi}$. along the creek, temp. $20^{\circ}$, strong $E$ wind; 8 species, 117 individuals. (Add: Ruffed Grouse, 14; Sharp-tailed Grouse, 6, Dec. 27; Hairy Woodpecker, 1; Black-billed Magpie, 1, Dec. 24: Common Raven, 7, Dec. 27). - Christine Pike (compiler), Sheila and Tommy Lamont.

WAUCHOPE, Dec. 24:21 mi. by car in $3 \mathrm{hrs}$ and $3 \mathrm{mi}$. on foot in $2-1 / 2 \mathrm{hrs}$ plus $1-1 / 2 \mathrm{hrs}$ about yard, temp. $10^{\circ}$ to $22^{\circ}$, wind S $15-0$ mph, 4 in. snow: 9 species, 673 individuals. (Add: Gray Partridge, 4, Dec. 30; Boreal Chickadee, I, Dec. 27; Evening Grosbeak, 11, Dec. 31; Common Redpoll, 3, Jan. 1), Dale Hjertaas.

WHITE BEAR, Dec. 27; temp. $30^{\circ}$, cloudy, no wind, little snow, $4 \mathrm{mi}$. by car and $3 \mathrm{mi}$. on foot: 3 species, 56 individuals. (Add: Evening Grosbeak, 6, Dec. 21) - Leroy
Clark, David and Douglas Gunn, Gerald, Laine, Gary and Sig Jordheim.

WYNYARD, Dec. $24: 47 \mathrm{mi}$. by car and around yard, temp. $10^{\circ}$ : calm, light overcast: 9 species, 558 individuals. (Add: Hairy Woodpecker, 1, Dec. 17) - John Gulley, Sherry Kachur.

YELLOW CREEK, Dec. 26; $6 \mathrm{mi}$. by car and $2 \mathrm{mi}$. on foot in $3 \mathrm{hrs}$, temp. $30^{\circ}$, clear, no wind, 6 in. snow; 10 species, 607 individuals, - Gaylene Mazur, Rosemary Nemeth.

STETTLER, Alta. Dec. 24; $40 \mathrm{mi}$. by car, 3 hrs. by snow shoe and around yard, temp. $22^{\circ}$ : partly sunny, calm, 6 in. snow; 10 species, 139 individuals. Mallard, 31; Bald Eagle, 1; Pileated Woodpecker, 1; Hairy Woodpecker, 1: Blue Jay, 2; Black-billed Magpie, 13; Black-capped Chickadee, 11: House Sparrow, 75: Evening Grosbeak, 3; Pine Grosbeak, 1. (Add: Goshawk, 1, Dec. 26: Golden Eagle, 1, Dec. 18; Ruffed Grouse, 2, Dec. 27: Sharp-tailed Grouse, 2, Dec. 21; Gray Partridge, 6, Dec. 29: Great Horned Owl, 1, Dec. 27: Snowy Owl, 1, Dec. 19: Downy Woodpecker, 1, Dec. 17: Snow Bunting, 200, Dec. 22). - Graeme Greenlee, Pat Greenlee, Lloyd Lohr (compiler) Lyman Matthews.

FORT SMITH, N.W.T., Dec. 27: $50 \mathrm{mi}$. by car in 3 hrs., temp $-5^{\circ}$; overcast with light snow falling, calm, $1-1 / 2$ feet of snow: 7 species 227 individuals. Rock Dove, 30: Great Gray Owl, 1: Gray Jay, 2: Common Raven, 99; Starling, 1; House Sparrow, 14; Common Redpoll, 80. (Add: Pine Grosbeak, 11. Dec. 21). - Ernie, Elsie, Pamela and Jonathan Kuyt.

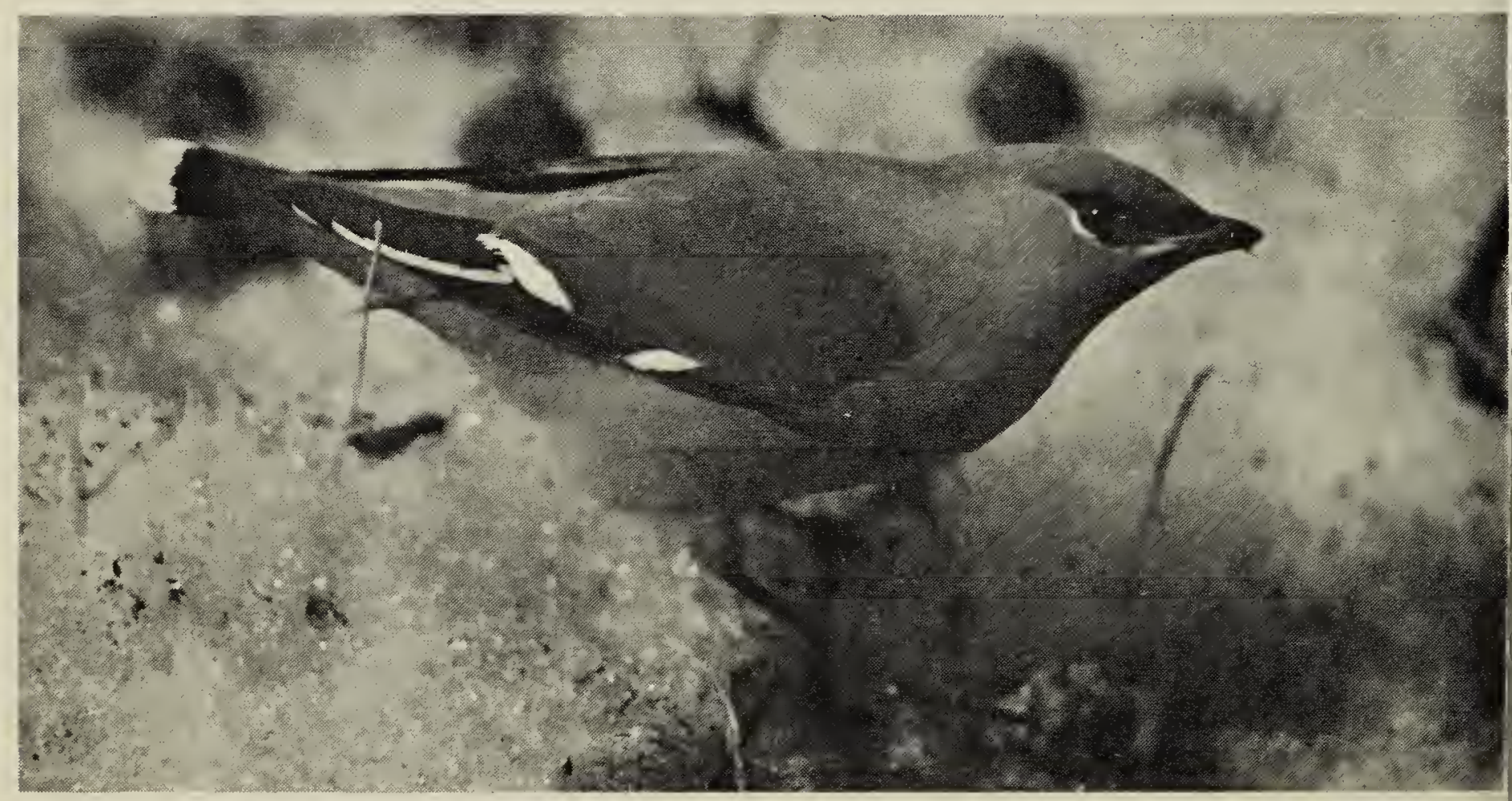

